\title{
Root Reconstruction with Total Replacement of Ascending Aorta Using Hypothermic Circulatory Arrest and Selective Cerebral Perfusion for Moderately Dilated Distal Ascending Aorta
}

\author{
Satoshi Yamashiro", Ryoko Arakaki, Yuya Kise, Hitoshi Inafuku, Yukio Kuniyoshi \\ Department of Thoracic and Cardiovascular Surgery, University of the Ryukyus, Nishihara, Okinawa, Japan \\ Email: *y3104@med.u-ryukyu.ac.jp
}

Received June 3, 2013; revised July 3, 2013; accepted July 10, 2013

Copyright (c) 2013 Satoshi Yamashiro et al. This is an open access article distributed under the Creative Commons Attribution License, which permits unrestricted use, distribution, and reproduction in any medium, provided the original work is properly cited.

\begin{abstract}
Objective: We have occasionally encountered a moderately dilated distal ascending aorta while reconstructing an aortic root. We describe reconstruction of an extended root and ascending aorta using our current strategy. Patients and Methods: Between March 2011 and December 2012, 15 (11 men; mean age, $70.1 \pm 7.3$ years) patients underwent root reconstruction with hemi-arch replacement under hypothermic circulatory arrest with antegrade selective cerebral perfusion. The maximum diameter of the aortic root was $52.5 \pm 4.4 \mathrm{~mm}$. The distal ascending aorta just below innominate artery was moderately dilated to $41.7 \pm 1.4 \mathrm{~mm}$ in diameter. Results: Operative outcomes excluding the diameter of the distal ascending aorta did not significantly differ from those of patients who had undergone root reconstruction under distal ascending aortic clamping during the same period. Postoperative computed tomography confirmed complete resection of the dilated ascending aorta in the patients, and did not develop neurological dysfunction or stroke. Conclusion: Postoperative computed tomography confirmed complete resection of dilated ascending aortae. We considered that complete resection under hypothermic circulatory arrest and selective cerebral perfusion might help to avoid repeated surgery to treat dilation of the distal ascending aorta over the long-term.
\end{abstract}

Keywords: Annulo-Aortic Ectasia; Re-Operation

\section{Introduction}

Ascending aorta replacement is the most common strategy for treating thoracic aortic pathologies. Graft replacement for an ascending aortic aneurysm is now a relatively safe and standard surgical procedure. However, management of a mildly to moderately dilated ascending aorta during cardiac surgery remains controversial. We have encountered some patients with a moderately dilated distal ascending aorta during aortic root reconstruction. Distal anastomosis of the ascending aorta usually proceeds under aortic clamping, when a dilated distal ascending aorta would remain. The most important consequence of an enlarged ascending aortic dimension is a proportional increase in the incidence of aortic rupture, dissection and re-operation. We have routinely wrapped borderline dilated distal ascending aortae with prosthetic grafts or felt strips to prevent dilation over the long term.

"Corresponding author.
However, we have recently replaced moderately dilated ascending aortae (hemi-arch replacement), using open distal anastomosis for complete resection.

\section{Patients and Methods}

Between March 2011 and December 2012, 15 patients (11 men and 4 women; mean age, $70.1 \pm 7.3$ years) underwent root reconstruction with total replacement of a dilated ascending aorta under hypothermic circulatory arrest (HCA) with antegrade selective cerebral perfusion (ASCP), (HCA group). The ascending aorta was completely resected just below the innominate artery (hemi-arch group). All patients were preoperatively diagnosed by computed tomography (CT), angiography and ultrasound cardiography as having annulo-aortic ectasia (AAE) with aortic regurgitation (AR). The maximum aortic root diameter was $52.5 \pm 4.4 \mathrm{~mm}$. The distal ascending aorta just below the innominate artery was moderately dilated 
to $41.7 \pm 1.4 \mathrm{~mm}$ in diameter. The root was reconstructed using the modified Bentall $(n=11)$ and David $(n=4)$ procedures.

The cerebral circulation of all patients undergoing elective surgery was preoperatively evaluated by Doppler ultrasonography of the extracranial vessels and by digital subtraction angiography of the extracranial and intracranial circulation. A cardiopulmonary bypass (CPB) was established with ascending aortic and right axillary arterial cannulation for arterial inflow and right atrial drainage. Core cooling was started during CPB to generate hypothermia over a period of 30 - 40 minutes, during which, the proximal repair proceeded. The circulation was arrested when the electroencephalogram flat-lined. With the patient in the Trendelenberg position, $12 \mathrm{Fr}$ malleable perfusion cannulae connected to an oxygenator by a separate single-roller pump head were inserted into the left common carotid and left subclavian arteries through the aortic lumen. The cannula in the right axillary artery was used for right-sided ASCP with proximal brachio-cephalic artery clamping using another singleroller pump. Cerebral perfusion was started at a rate of $10-15 \mathrm{~mL} / \mathrm{min} / \mathrm{kg}$ and adjusted to maintain left radial arterial pressure and bilateral carotid arterial stump pressure between 40 and $60 \mathrm{mmHg}$. Perioperative blood flow through the middle cerebral arteries was continuously monitored using bilateral transcranial Doppler (Viasys Inc. Conshocken, PA, USA) ultrasonography. Cerebral oxygen saturation was monitored using an Invos ${ }^{\circledR}$ cerebral oximeter (Somanetics Co., Troy, MI, USA). Distal repair proceeded via open distal anastomosis with a single-branched Dacron graft. Both HCA and ASCP were terminated after reconstructing the distal ascending aorta with antegrade perfusion from the graft branch and then the proximal repair was completed during rewarming. Finally, the proximal and distal grafts were anastomosed.

We compared the surgical results with those of patients whose root reconstruction had proceeded under distal ascending aortic clamping during the same period (Clamp group). We also compared postoperative CT findings with those of patients who had a moderately dilated distal ascending aorta that had been wrapped.

Continuous data are expressed as means $\pm \mathrm{SD}$, and categorical variables are expressed as ratios (\%). Characteristics were compared between the two groups using Student's t-test.

\section{Results}

The durations of surgery, total CPB, cardiac ischemia, ASCP and HCA were 445.6 \pm 42.7, $239.9 \pm 30.9,211.3$ \pm 28.2 , $33.9 \pm 5.9$ and $33.0 \pm 5.1$ minutes, respectively, in the HCA group. Rectal temperature in these patients was maintained at $25.4^{\circ} \mathrm{C} \pm 1.6^{\circ} \mathrm{C}$ during HCA. Operative outcomes excluding the diameter of the distal ascending aorta $(41.5 \pm 1.4$ vs. $35.4 \pm 2.8 \mathrm{~mm})$ and the lowest rectal temperature during operation (25.4 \pm 1.6 vs. $30.6^{\circ} \mathrm{C} \pm 3.7^{\circ} \mathrm{C}$ ) did not significantly differ from those of the Clamp group (Table 1).

Neurological dysfunction and postoperative stroke did not arise in this series. Mechanical ventilation was re-

Table 1. Patient's characteristics and operative outcomes.

\begin{tabular}{|c|c|c|c|}
\hline Group & HCA group $(n=15)$ & Clamp group $(\mathrm{n}=8)$ & $P$ value \\
\hline Age (years old) & $70.1 \pm 7.3$ & $65.5 \pm 13.9$ & \\
\hline Gender (M/F) & $11 / 4$ & $7 / 1$ & \\
\hline Max Asc Ao. diameter (mm) & $52.0 \pm 5.7$ & $49.8 \pm 10.4$ & \\
\hline Distal Asc Ao. diameter (mm) & $41.5 \pm 1.4$ & $35.4 \pm 2.8$ & $P=0.04$ \\
\hline Surgical duration (min) & $445.6 \pm 42.7$ & $505.9 \pm 131.6$ & \\
\hline CPB time (min) & $239.9 \pm 31.9$ & $253.4 \pm 73.6$ & \\
\hline Cardiac arrest time (min) & $211.3 \pm 28.7$ & $217.4 \pm 67.0$ & \\
\hline Bleeding (ml) & $692.2 \pm 298.2$ & $769.0 \pm 454.6$ & \\
\hline Blood Transfusion (ml) & $2017.7 \pm 698.0$ & $1965.0 \pm 1021.0$ & \\
\hline Rectal temp $\left({ }^{\circ} \mathrm{C}\right)$ & $25.4 \pm 1.6$ & $30.6 \pm 3.7$ & $P=0.04$ \\
\hline Respiratory time (hours) & $12.2 \pm 4.2$ & $13.8 \pm 5.0$ & \\
\hline Morbidity & 0 & 1 ; respiratory failure (12.5\%) & \\
\hline Hospitalization (POD; days) & $26.2 \pm 5.9$ & $35.6 \pm 15.1$ & \\
\hline
\end{tabular}

HCA group: patients underwent root reconstruction with total replacement of the ascending aorta under hypothermic circulatory arrest (HCA) with antegrade selective cerebral perfusion (ASCP); Clamp group: patients proceeded root reconstruction under distal ascending aortic clamping; Asc Ao.: ascending aorta; CPB: cardiopulmonary bypass; POD: post-operative days. 
quired after surgery for $12.2 \pm 4.2$ hours. Postoperative CT confirmed complete resection of the dilated ascending aorta (Figures 1 and 2). However, the distal ascending aorta clearly remained dilated after clamping (previous patients; Figure 3). All patients remained in hospital for $26.2 \pm 5.9$ days after surgery. Aetiology of aortic aneurysm of this series were not significant different between both groups (Table 2).

\section{Discussion}

Although the results of surgical intervention of the thoracic aorta have gradually improved, hospital mortality rates as well as neurological and systemic complications remain considerable [1]. Therefore, management of the mildly to moderately dilated ascending aorta has remained controversial. In general, surgery with concomitant cardiovascular procedures especially involving the

Table 2. Aetiology of aneurysms.

\begin{tabular}{ccc}
\hline & HCA group & Clamp group \\
\hline Atherosclerotic change & $8(53.3 \%)$ & $4(50.0 \%)$ \\
Degenerative change & $6(40.0 \%)$ & $3(37.5 \%)$ \\
Central medial necrosis & $2(13.3 \%)$ & $1(12.5 \%)$ \\
Aortitis & $1(6.7 \%)$ & $1(12.5 \%)$ \\
Bicuspid valve & $1(6.7 \%)$ & 0 \\
Marfan syndrome & 0 & $1(12.5 \%)$ \\
\hline
\end{tabular}



Figure 1. Postoperative computed tomography image after hemi-arch replacement with hypothermic circulatory arrest and antegrade selective cerebral perfusion. Dilated ascending aorta is completely resected (arrow).

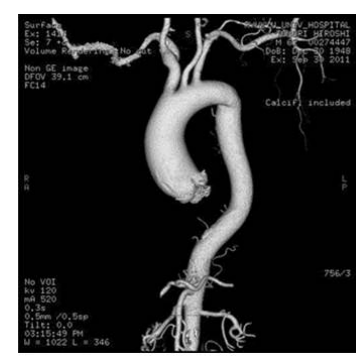

(a)

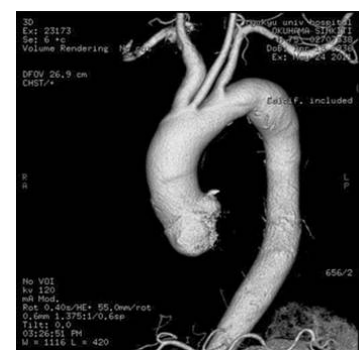

(c)
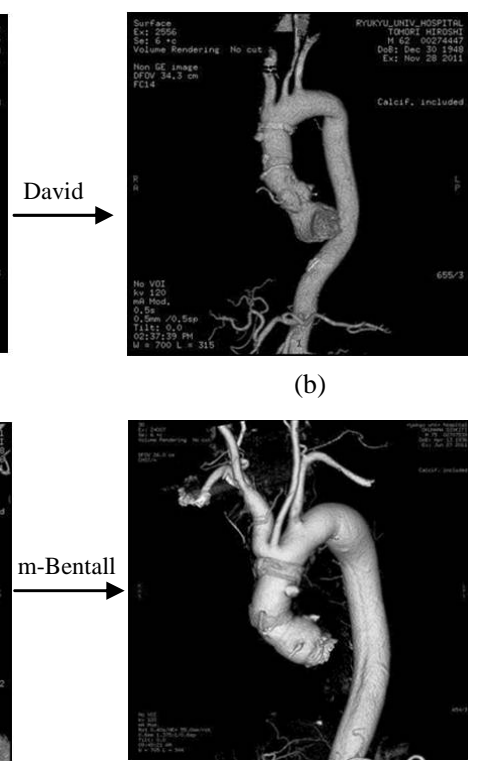

(d)
Figure 2. Postoperative computed tomography image after hemi-arch replacement with hypothermic circulatory arrest and antegrade selective cerebral perfusion. Dilated ascending aortae are completely resected after David procedure (a, c) and modified-Bentall operation (b, d) (arrows).



(a)

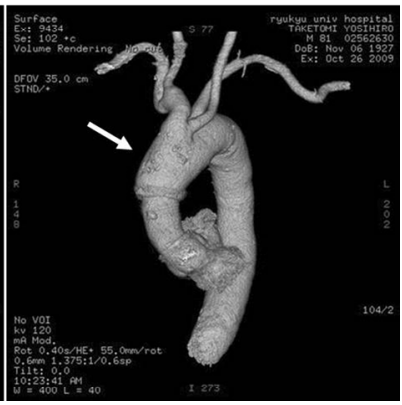

(b)
Figure 3. Postoperative three-dimensional computed tomography image. (a, b) Distal ascending aorta remains dilated after clamping for distal anastomosis of ascending aorta (arrows).

aortic valve can proceed when aortic dilation is between $45-50 \mathrm{~mm}$ in diameter. We had previously wrapped borderline dilated distal ascending aortae with a felt or prosthetic graft to prevent dilation over the long term. Some reports have indicated that wrapping effectively preserves dilated ascending aortae [2-4]. However, we recently experienced a need to re-operate on a replaced distal ascending aorta of a male patient that had become dilated despite being wrapped with a prosthetic graft (Figure 4). The distal ascending aorta at the initial operation was moderately dilated to a diameter of $43 \mathrm{~mm}$. Thereafter, we considered that extensive replacement of the dilated ascending aorta (hemi-arch replacement) using open distal anastomosis might have been a better 


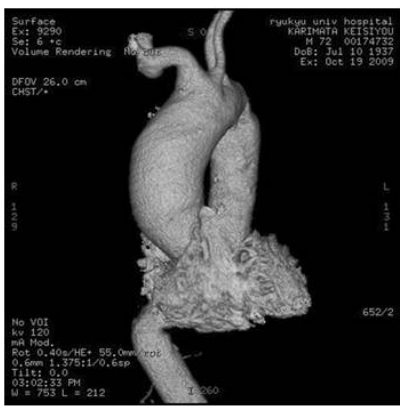

(a)

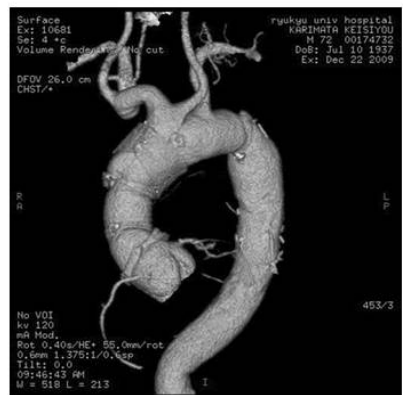

(b)

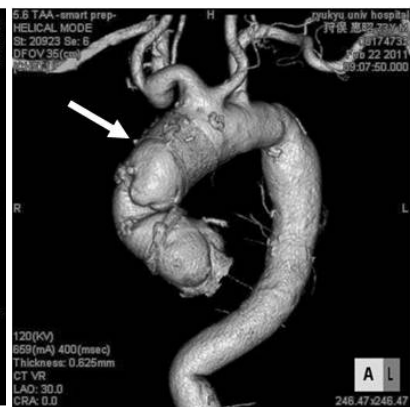

(c)

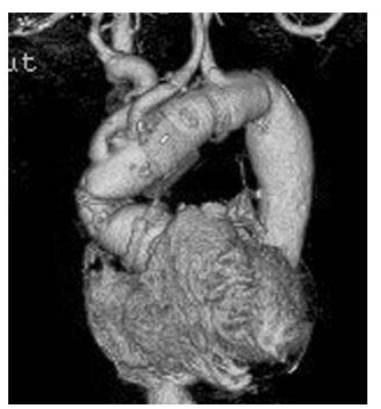

(d)
Figure 4. Pre- and post-operative computed tomography images. (a) Aneurysmal dilation of ascending aorta to maximal diameter of $53 \mathrm{~mm}$ in a patient with mildly dilated distal ascending aorta; (b) Borderline dilated distal ascending aorta wrapped with felt; (c) Distal ascending aorta includes arch aorta enlarged (arrow) at one year after initial operation; (d) Reconstructed vessel after repeated aortic arch replacement.

choice.

Over the past 2 decades, various adjunctive techniques of cerebral circulatory management have evolved to increase the safety and duration of cerebral protection. These techniques include HCA alone, retrograde cerebral perfusion (RCP) with HCA, and ASCP with HCA. The safe time period of HCA to prevent temporary neurologic dysfunction has been reported to be less than 45 minutes [5]. The safe duration of HCA is limited and prolonged periods of HCA are associated with end-organ ischemia and postoperative neurologic dysfunction [6]. Therefore, further efforts to improve cerebral protection have led to the development of antegrade and retrograde cerebral perfusion. The optimal method of cerebral perfusion (antegrade versus retrograde) is a well-debated topic and has yet to be determined. RCP can be instituted easily just by clamping the venous drainage tube of the inferior vena cava, snaring the superior vena cava cannula, and counter-rotating the CPB pump. However, this method is associated with two major concerns: insufficient cerebral oxygen supply and cerebral edema with increased intracranial pressure due to high venous pressure [7]. ASCP is employed in many institutions but requires extra arterial cannulation and, in some cases, an extra arterial pump; thus, it is relatively complicated and time-consuming [8]. However, only about $20 \%$ of adults have anatomically and functionally completed circle of Wills [9]. Therefore, we considered that bilateral ASCP might be unachievable via unilateral carotid perfusion.

Both HCA with ASCP are currently being applied to protect the brain. Svenson et al. [10] started applying a subclavian or axillary artery cannulation strategy with HCA and antegrade brain perfusion in 1995, and found that it was a safe approach to aortic arch surgery because the stroke risk was $<2 \%$. Technical simplicity and avoidance of aorta and arch vessel manipulation, as well as a bloodless operative field, render DHCA an alternative method of brain protection, especially during ascending aorta hemi-arch replacement, when a short period of circulatory arrest is anticipated. The advantages of ASCP are that circulatory arrest can be safely extended up to 90 minutes, which allows more complex aortic repair [11]. However, hypothermia-associated coagulopathy and pulmonary, renal and micro embolic complications have been cited in the literature as important disadvantages [11]. Rectal temperature in our series was maintained at $25^{\circ} \mathrm{C}$ during HCA and we did not find an increased risk of coagulative complications. Moderate, instead of profound hypothermia can reduce the risk of coagulative and systemic complications. The degree of hypothermia during this procedure should be considered when a short period of circulatory arrest is anticipated. Cerebral oximetry is a functional and possibly superior method of monitoring cerebral perfusion and oxygenation [12,13]. Although cerebral protection techniques are continually undergoing refinement with improved results, we added left brain perfusion to minimize the likelihood of associated brain damage arising [14]. Moreover, vertebral perfusion via the left axillary artery is important for spinal, as well as cerebral protection. Therefore, we suppose that our method of cerebral protection is acceptable. The optimal approach to cerebral protection during these procedures remains controversial.

Indications for total arch replacement in patients with mildly to moderately dilated ascending and arch aortae are contentious because the surgical results of extended arch replacement are suboptimal [15]. Postoperative CT revealed complete resection of the dilated ascending aorta using our procedures. We considered that such complete resection under HCA and ASCP might prevent the need for re-operation to treat a dilated distal ascending aorta over the long term. Neither permanent neurological dysfunction nor postoperative stroke developed in this series. Our results suggested that extensive reimplantation of the ascending aorta accompanied by adequate HCA and ASCP is effective. Although this study is limited by the relatively small patient cohort, our findings might have practical implications for the evolution 
of controversial cases. Aetiology of aortic aneurysm of this series was not significant different between both groups. We considered that it is important to the distal aortic diameter.

\section{REFERENCES}

[1] M. A. Ergin, D. Spielvogel, A. Apaygin, S. L. Lansman, J. N. McCullough, J. D. Galla and R. B. Griepp, "Surgical Treatment of the Dilated Ascending Aorta: When and How?” The Annals of Thoracic Surgery, Vol. 67, No. 6, 1999, pp. 1834-1839.

doi:10.1016/S0003-4975(99)00439-7

[2] O. Cohen, J. Odim, D. D. L. Zerda, C. Ukatu, R. Vyas, N. Vyas, K. Oaratnik and H. Laks, "Long Term Experience of Girdling the Ascending Aorta with Dacron Mesh as Definitive Treatment for Aneurismal Dilatation," The Annals of Thoracic Surgery, Vol. 83, No. 2, 2007, pp. S780-S784. doi:10.1016/j.athoracsur.2006.10.086

[3] G. I. Tagarakis, D. Karangelis, A. Baddour, M. E. Daskalopouls, V. T. Liouras, D. Papadopoulos, K. Stamoulis, S. S. Lampoura and N. B. Tsilimingas, "An Alternate Solution for the Treatment of Ascending Aortic Aneurysms: The Wrapping Technique," Journal of Cardiothoracic Surgery, Vol. 5, 2010, p. 100. doi:10.1186/1749-8090-5-100

[4] E. Tappainer, V. Fiorani, A. Nocchi, E. Likaj, S. Memishaj and M. Zogno, "Safe Wrapping of the Borderline Dilated Ascending Aorta during Aortic Valve Replacement," Journal of Cardiothoracic Surgery, Vol. 2, 2007, p. 15. doi:10.1186/1749-8090-2-15

[5] A. Gega, J. A. Rizzo, M. H. Johnson, M. Tranquilli, E. A. Farkas and J. A. Elefteriades, "Straight Deeo Hypothermic Arrest: Experience in 394 Patients Supports Its Effectiveness as a Sole Means of Brain Preservation,” The Annals of Thoracic Surgery, Vol. 84, No. 3, 2007, pp. 759-767. doi:10.1016/j.athoracsur.2007.04.107

[6] M. A. Ergin, J. D. Galla, S. L. Lansman, C. Quintana, C. Bodian and R. B. Gripp, "Hypothermic Circulatory Arrest in Operations on the Thoracic Aorta. Determinants of Operative Mortality and Neurologic Outcome,” The Journal of Thoracic and Cardiovascular Surgery, Vol. 107, No. 3, 1994, pp. 788-797.

[7] A. Usui, K. Oohara, T. L. Liu, M. Murase, M. Tanaka, E. Takeuchi and T. Abe, "Determination of Optimum Retrograde Cerebral Perfusion Conditions," The Journal of Thoracic and Cardiovascular Surgery, Vol. 107, No. 1, 1994, pp. 300-308.

[8] H. Ogino, H. Sasaki, K. Minatoya, H. Matsuda, H. Ta- naka, H. Watanuki, M. Ando and S. Kitamura, "Evolving Arch Surgery Using Integrated Selective Cerebral Perfusion: Impact of Axillary Artery Perfusion,” The Journal of Thoracic and Cardiovascular Surgery, Vol. 136, No. 3, 2008, pp. 641-648. doi:10.1016/j.jtcvs.2008.02.089

[9] S. Morita, M. Yasaka, K. Yasumori, Y. Oishi, T. Takaseya, H. Sonoda and T. Kawara, "Transcranial Doppler Study to Assess Intracranial Arterial Communication before Aortic Arch Operation," The Annals of Thoracic Surgery, Vol. 86, No. 2, 2008, pp. 448-451. doi:10.1016/j.athoracsur.2008.04.024

[10] L. G. Svenson, E. M. Nadolny, D. L. Penney, J. Jacobson, W. A. Kimmel, M. H. Entrup and R. S. D’Agostino, "Prospective Randomized Neurocognitive and S-100 Study of Hypothermic Circulatory Arrest, Retrograde Brain Perfusion, and Antegrade Brain Perfusion for Aortic Arch Operations,” The Annals of Thoracic Surgery, Vol. 71, No. 6, 2001, pp. 1905-1912. doi:10.1016/S0003-4975(01)02570-X

[11] M. Eusanio, R. M. J. Wesselink, W. Morsunis, K. M. Dossche and M. A. A. M. Schpens, "Deep Hypothermic Circulatory Arrest and Antegrade Selective Cerebral Perfusion during Ascending Aorta-Hemiarch Replacement: A Retrospective Comparative Study," The Journal of Thoracic and Cardiovascular Surgery, Vol. 125, No. 4, 2003, pp. 849-854. doi:10.1067/mtc.2003.8

[12] N. Fassiadis, H. Zayed, H. Rashid and D. W. Green, "Invos Cerebral Oximeter Compared with the Transcranial Doppler for Monitoring Adequacy of Cerebral Perfusion in Patients Undergoing Carotid Endarterectomy," International Journal of Angiology, Vol. 25, 2006, pp. 401406.

[13] S. K. Samra, E. A. Dy, K. Welch, P. Dorje, G. B. Zelenock and J. C. Stanley, "Evaluation of a Cerebral Oximeter as a Monitor of Cerebral Ischemia during Carotid Endarterectomy,” Anesthesiology, Vol. 93, No. 4, 2000, pp. 964-970. doi:10.1097/00000542-200010000-00015

[14] S. Yamashiro, Y. Kuniyoshi, K. Arakaki, H. Inafuku, Y. Morishima and Y. Kise, "Total Arch Replacement Using Bilateral Axillary Antegrade Selective Cerebral Perfusion,” Annals of Thoracic and Cardiovascular Surgery, Vol. 16, No. 4, 2010, pp. 216-219.

[15] R. Codecasa, M. A. Mariani, A. D’Alfonso, C. Nardi and J. G. Grandjean, “Current indications for Elective Surgical Treatment of Dilated Ascending Aorta: A New Formula," Annals of Thoracic and Cardiovascular Surger, Vol. 125, No. 6, 2003, pp. 1528-1530. doi:10.1016/S0022-5223(03)00059-X 